\title{
ON THE POWER-COMMUTATIVE KERNEL OF LOCALLY NILPOTENT GROUPS
}

\author{
COSTANTINO DELIZIA AND CHIARA NICOTERA
}

Received 5 July 2005

We define the power-commutative kernel of a group. In particular, we describe the powercommutative kernel of locally nilpotent groups, and of finite groups having a nontrivial center.

A group $G$ is called power commutative, or a PC-group, if $\left[x^{m}, y^{n}\right]=1$ implies $[x, y]=1$ for all $x, y \in G$ such that $x^{m} \neq 1, y^{n} \neq 1$. So power-commutative groups are those groups in which commutativity of nontrivial powers of two elements implies commutativity of the two elements. Clearly, $G$ is a $P C$-group if and only if $C_{G}(x)=C_{G}\left(x^{n}\right)$ for all $x \in G$ and all integers $n$ such that $x^{n} \neq 1$. Obvious examples of $P C$-groups are groups in which commutativity is a transitive relation on the set of nontrivial elements (CT-groups) and groups of prime exponent.

Recall that aroup $G$ is called an $R$-group if $x^{n}=y^{n}$ implies $x=y$ for all $x, y \in G$ and for all positive integers $n$. In other words, $R$-groups are groups in which the extraction of roots is unique. A result due to Mal'cev and Cernikov (see, e.g., [3]) states that every nilpotent torsion-free group is an $R$-group. There is a natural connection between $P C$ groups and $R$-groups. For, as pointed out in [3], a torsion-free group is a $P C$-group if and only if it is an $R$-group.

In [5], Wu gave the classification of locally finite $P C$-groups. In particular, she proved that a finite group is a $P C$-group if and only if the centralizer of each nontrivial element is abelian or of prime exponent. This result implies that a finite group having a nontrivial center is a $P C$-group if and only if it is abelian or it has prime exponent. Moreover, the class of $P C$-groups is contained in the class of groups in which the centralizer of each nontrivial element is nilpotent. This class of groups was investigated by many authors (see, e.g., $[1,4])$.

In analogy to what is done in [2] to define the commutative-transitive kernel of a group, we introduce an ascending series

$$
\{1\}=P_{0}(G) \leq P_{1}(G) \leq \cdots \leq P_{t}(G) \leq \cdots
$$

of characteristic subgroups of $G$ contained in the derived subgroup $G^{\prime}$. We define $P_{1}(G)$ as 
the subgroup of $G^{\prime}$ generated by those commutators $[x, y]$ such that there exist positive integers $n, m$ with $x^{n} \neq 1, y^{m} \neq 1$, and $\left[x^{n}, y^{m}\right]=1$. If $t>1$ then $P_{t}(G)$ is defined by $P_{t}(G) / P_{t-1}(G)=P_{1}\left(G / P_{t-1}(G)\right)$. Finally, the PC-kernel of $G$ is the subgroup $P(G)$ of $G^{\prime}$ defined by

$$
P(G)=\bigcup_{t \in \mathbb{N}} P_{t}(G)
$$

Obviously, for any group $G$, the $P C$-kernel $P(G)$ is characteristic in $G, G / P(G)$ is a $P C$ group, and $G$ is a $P C$-group if and only if $P(G)=\{1\}$.

Let $\mathscr{X}$ be a class of groups. Then one can ask whether there exists a nonnegative integer $n$ such that $P_{n}(G)=P(G)$ for all $G \in \mathscr{X}$. Of course $P(G)=P_{n}(G)$ if and only if $G / P_{n}(G)$ is a $P C$-group.

In this paper, we give affirmative answers to the previous question when $\mathscr{X}$ is the class of locally nilpotent groups, or the class of finite groups having a nontrivial center. In both cases, we prove that $P(G)=P_{1}(G)$ for all $G \in \mathscr{X}$.

Our first results are concerned with the power-commutative kernel of finite nilpotent groups.

Proposition 1. Let $p$ be a prime and $G$ a finite p-group. Then $G / P_{1}(G)$ is a PC-group.

Proof. Notice that $P_{1}(G) \leq M$ for every maximal subgroup $M$ of $G$ since $P_{1}(G) \leq G^{\prime} \leq$ $\Phi(G)$, where $\Phi(G)$ is the Frattini subgroup of $G$. This implies that $M / P_{1}(G)$ is a maximal subgroup of $G / P_{1}(G)$ if and only if $M$ is a maximal subgroup of $G$.

Let $G$ be a counterexample of least order. For any maximal subgroup $M$ of $G$ we obtain $M / P_{1}(G) \simeq\left(M / P_{1}(M)\right) /\left(P_{1}(G) / P_{1}(M)\right)$. Hence $M / P_{1}(G)$ is a $P C$-group since it is a quotient of a finite $P C$-group (see [5]). It follows that a maximal subgroup of $G / P_{1}(G)$ is abelian or it has exponent $p$.

Put $\bar{G}=G / P_{1}(G)$ and $\bar{H}=H / P_{1}(G)$ for all $P_{1}(G) \leq H \leq G$. If every maximal subgroup $\bar{M}$ of $\bar{G}$ has exponent $p$, then $G$ is cyclic or of exponent $p$. In any case $\bar{G}$ is a $P C$-group, that is a contradiction. So we may assume that $\bar{G}$ has a maximal subgroup $\bar{M}$ such that $\bar{M}$ is abelian and $\bar{M}^{p} \neq 1$. Consider $g \in \bar{G} \backslash \bar{M}$, so $\bar{G}=\langle\bar{M}, g\rangle$. Moreover $|\bar{G}: \bar{M}|=p$.

If there exists $a \in \bar{M}$ such that $(g a)^{p} \neq 1$, then $(g a)^{p} \in \bar{M} \backslash\{1\}$. So, for all $y \in \bar{M}$ we get $\left[y,(g a)^{p}\right]=1$, hence $[y, g]=[y, g a]=1$. It follows that $\bar{G}$ is abelian, a contradiction. Thus $(g a)^{p}=1$ for all $a \in \bar{M}$, and in particular $g^{p}=1$. It follows that $a^{g^{p-1}+\cdots+g+1}=(g a)^{p}=$ 1 for all $a \in \bar{M}$. This implies $a^{p}=1$ for all $a \in C_{\bar{M}}(g)$, so $\left(C_{\bar{M}}(g)\right)^{p}=C_{\bar{M}^{p}}(g)=1$. But $\bar{M}^{p} \cap Z(\bar{G}) \neq 1$ since $\bar{M}^{p} \neq 1$, that is a contradiction.

Proposition 2. Let $G$ be a finite nilpotent group of order $n=p_{1}^{\alpha_{1}} \cdots p_{t}^{\alpha_{t}}\left(p_{1}, \ldots, p_{t}\right.$ distinct primes). If $t>1$ then $G / P_{1}(G)$ is abelian.

Proof. Let $G_{p_{i}}$ be the Sylow $p_{i}$-subgroup of $G$ for all $i \in\{1, \ldots, t\}$; we will prove that $\left(G_{p_{i}}\right)^{\prime} \leq P_{1}(G)$ for all $i \in\{1, \ldots, t\}$. Let $x, y \in G_{p_{i}} \backslash\{1\}, a \in G_{p_{1}} \times \cdots \times G_{p_{i-1}} \times G_{p_{i+1}} \times$ $\cdots \times G_{p_{t}}$. Put $|a|=m$ and $|x|=p_{i}{ }^{r}$. Now $|a x|=m p_{i}{ }^{r}$ as $\left(m, p_{i}{ }^{r}\right)=1$. Since $(a x)^{p_{i}{ }^{r}}=a^{p_{i}{ }^{r}}$ has order $m$ we get $\left[(a x)^{p_{i}{ }^{r}}, y\right]=\left[a^{p_{i}{ }^{r}}, y\right]=1$. Thus $[a x, y]=[x, y] \in P_{1}(G)$.

Corollary 3. Let $G$ be a finite nilpotent group; then $G / P_{1}(G)$ is abelian or it has exponent p. In both cases $G / P_{1}(G)$ is a PC-group. 
Proof. The result is an immediate consequence of the previous propositions and [5, Theorem 4].

Now we prove that the equality $P(G)=P_{1}(G)$ holds for every nilpotent group $G$.

Theorem 4. Let $G$ be a nilpotent group. Then $G / P_{1}(G)$ is a PC-group.

Proof. If $G$ is torsion-free then $G$ is a $P C$-group (see [3]), so $P_{1}(G)=\{1\}$ and the result is true. So we may suppose that the torsion subgroup $T$ of $G$ is nontrivial.

First of all, notice that if for elements $x, y \in G \backslash\{1\}$ there exists a positive integer $n$ such that $x^{n} \neq 1$ and $\left[x^{n}, y\right]=1$, then $[x, y] \in T$. This is obvious if $x \in T$ or $y \in T$, so we may assume $x, y \notin T$. Then $\langle x, y\rangle T / T \leq G / T$. So $\langle x T, y T\rangle$ is torsion-free, and $\left[(x T)^{n}, y T\right]=T$ implies $[x, y] \in T$. This means that $P_{1}(G) \subseteq T$.

If for any $x, y \in G$ the commutator $[x, y]$ is periodic, then it is easy to see that there exists a positive integer $m$ such that $\left[x, y^{m}\right]=1$. In fact, $\langle x, y\rangle$ is a $F C$-group since $\langle x$, $y\rangle / Z(\langle x, y\rangle)$ is finite, and therefore the set $\left\{x^{y^{t}} \mid t \in \mathbb{Z}\right\}$ is finite.

Now notice that if $x \in T$ then $[x, g] \in P_{1}(G)$ for all $g \in G \backslash T$. In fact, $[x, g] \in T$ implies that there exists a positive integer $m$ such that $\left[x, g^{m}\right]=1$. So we get $[x, g] \in P_{1}(G)$ because $g^{m} \neq 1$.

Finally, let $x, y \in G \backslash P_{1}(G)$ such that $x^{n} \notin P_{1}(G)$ and $\left[x^{n}, y\right] \in P_{1}(G)$. If $x, y \in T$ then $\langle x, y\rangle$ is a finite nilpotent group and Corollary 3 implies that $\langle x, y\rangle / P_{1}(\langle x, y\rangle)$ is a finite $P C$-group. Hence $\langle x, y\rangle / P_{1}(G) \cap\langle x, y\rangle$ is a $P C$-group and $[x, y] \in P_{1}(G)$. If $x \in T$ or $y \in$ $T$ then $[x, y] \in P_{1}(G)$, as noticed before. So we may suppose $x, y \in G \backslash T$. Since $\left[x^{n}, y\right] \in$ $P_{1}(G) \subseteq T$, we get $\left[x^{n}, y\right] \in T$ and so there exists a positive integer $m$ such that $\left[x^{n}, y^{m}\right]=$ 1. Therefore $[x, y] \in P_{1}(G)$, and the proof is complete.

Theorem 5. Let $G$ be a locally nilpotent group. Then $P(G)=P_{1}(G)$.

Proof. Let $x, y \in G \backslash P_{1}(G)$ such that $x^{n} \notin P_{1}(G)$ and $\left[x^{n}, y\right] \in P_{1}(G)$. Then

$$
\left[x^{n}, y\right]=\prod_{i=1}^{r}\left[a_{i}, b_{i}\right],
$$

where $a_{i}, b_{i} \in G$ for all $i=1,2, \ldots, r$, and $\left[a_{i}^{\alpha_{i}}, b_{i}^{\beta_{i}}\right]=1$ for some positive integers $\alpha_{i}$ and $\beta_{i}$ such that $a_{i}^{\alpha_{i}} \neq 1$ and $b_{i}^{\beta_{i}} \neq 1$.

Let $H=\left\langle x, y, a_{1}, \ldots, a_{r}, b_{1}, \ldots, b_{r}\right\rangle$. Then $H$ is nilpotent, so $H / P_{1}(H)$ is a $P C$-group by Theorem 4 . Since $\left[a_{i}, b_{i}\right] \in P_{1}\left(\left\langle a_{i}, b_{i}\right\rangle\right) \leq P_{1}(H)$ for all $i=1,2, \ldots, r$, we get $\left[x^{n}, y\right] \in$ $P_{1}(H)$. Thus $[x, y] \in P_{1}(H)$, and therefore $[x, y] \in P_{1}(G)$.

Now it is possible to prove that $P(G)=P_{1}(G)$ for any finite group $G$ such that $Z(G) \neq$ $\{1\}$.

Proposition 6. Let $G$ be a finite group such that $Z(G) \neq\{1\}$. Then $[a, b] \in P_{1}(G)$ for all $a, b \in G \backslash\{1\}$ such that $(|a|,|b|)=1$.

Proof. Put $|a|=n$ and $|b|=m$. Then there exists $z \in Z(G) \backslash\{1\}$ such that $|z|$ does not divide $n$ or $m$. Suppose $|z|$ does not divide $n$. Then $\left[(a z)^{n}, b\right]=\left[a^{n} z^{n}, b\right]=\left[z^{n}, b\right]=1$. Moreover $(a z)^{n}=z^{n} \neq 1$ and this yields $[a z, b]=[a, b] \in P_{1}(G)$. 


\section{On the power-commutative kernel of locally nilpotent groups}

Proposition 7. Let $G$ be a finite group such that $Z(G) \neq\{1\}$. Then $G / P_{1}(G)$ is nilpotent.

Proof. We may assume that the order of $G / P_{1}(G)$ is not a prime power. Let $p$ be any prime divisor of $\left|G / P_{1}(G)\right|$. Then $p$ divides $|G|$ and $P P_{1}(G) / P_{1}(G)$ is a Sylow $p$-subgroup of $G / P_{1}(G)$ whenever $P$ is a Sylow $p$-subgroup of $G$. We are going to show that $P P_{1}(G) / P_{1}(G)$ is normal in $G / P_{1}(G)$. Let $q \neq p$ be any prime dividing $\left|G / P_{1}(G)\right|$, and let $Q$ be a Sylow $q$-subgroup of $G$. Then $Q P_{1}(G) / P_{1}(G)$ centralizes $P P_{1}(G) / P_{1}(G)$, by Proposition 6 . Thus the normalizer in $G / P_{1}(G)$ of $P P_{1}(G) / P_{1}(G)$ contains a Sylow $q$-subgroup of $G / P_{1}(G)$ for all prime divisors of its order. Therefore this normalizer is actually $G / P_{1}(G)$, and the result follows.

Theorem 8. Let $G$ be a finite group such that $Z(G) \neq\{1\}$. Then $G / P_{1}(G)$ is abelian or it has exponent $p$.

Proof. Since $G / P_{1}(G)$ is nilpotent by Proposition 7, by [5] it suffices to show that $G / P_{1}(G)$ is a $P C$-group. Suppose not, and let $G$ be a counterexample of least order. We may assume $G$ is not nilpotent, hence $P_{1}(G) \npreceq \Phi(G)$. Thus there exists a maximal subgroup $M$ of $G$ such that $P_{1}(G) \nsubseteq M$. In particular $G^{\prime} \nsubseteq M$. If $Z(G) \nsubseteq M$, then there exists $z \in$ $Z(G) \backslash M$. Since $M$ is maximal, it follows that $\langle z\rangle M=G$. Hence $M$ is normal in $G$, and $G / M$ is cyclic. This in turn implies that $G^{\prime} \subseteq M$, a contradiction. Thus $Z(G) \subseteq M$, and so $Z(M) \neq\{1\}$. Then $M / P_{1}(M)$ is a $P C$-group and therefore $G / P_{1}(G) \simeq\left(M / P_{1}(M)\right) /((M \cap$ $\left.\left.P_{1}(G)\right) / P_{1}(M)\right)$ is a $P C$-group, the final contradiction.

\section{References}

[1] W. Feit, M. Hall Jr., and J. G. Thompson, Finite groups in which the centralizer of any nonidentity element is nilpotent, Math. Z. 74 (1960), 1-17.

[2] B. Fine, A. M. Gaglione, G. Rosenberger, and D. Spellman, The commutative transitive kernel, Algebra Colloq. 4 (1997), no. 2, 141-152.

[3] A. G. Kurosh, The Theory of Groups, Chelsea, New York, 1960.

[4] M. Suzuki, Finite groups with nilpotent centralizers, Trans. Amer. Math. Soc. 99 (1961), 425470.

[5] Y.-F. Wu, On locally finite power-commutative groups, J. Group Theory 3 (2000), no. 1, 57-65.

Costantino Delizia: Dipartimento di Matematica e Informatica, Università di Salerno, via Ponte don Melillo, 84084 Fisciano (SA), Italy

E-mail address: cdelizia@unisa.it

Chiara Nicotera: Dipartimento di Matematica e Informatica, Università di Salerno, via Ponte don Melillo, 84084 Fisciano (SA), Italy

E-mail address: cnicotera@unisa.it 


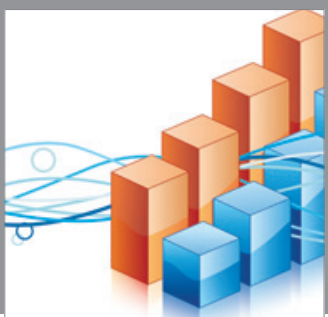

Advances in

Operations Research

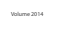

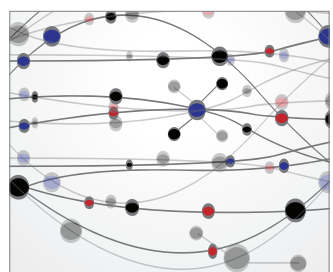

\section{The Scientific} World Journal
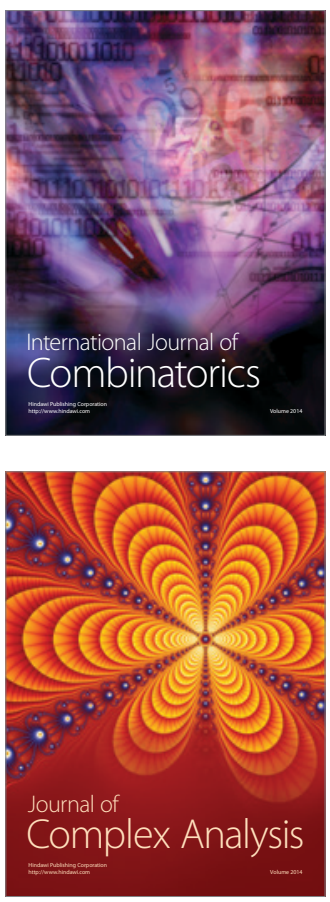

International Journal of

Mathematics and

Mathematical

Sciences
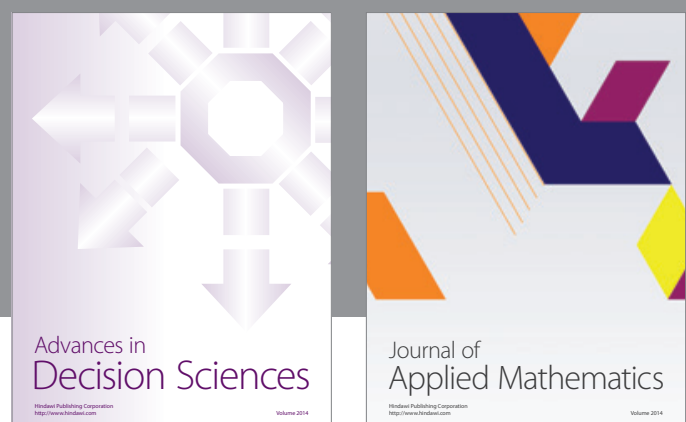

Journal of

Applied Mathematics
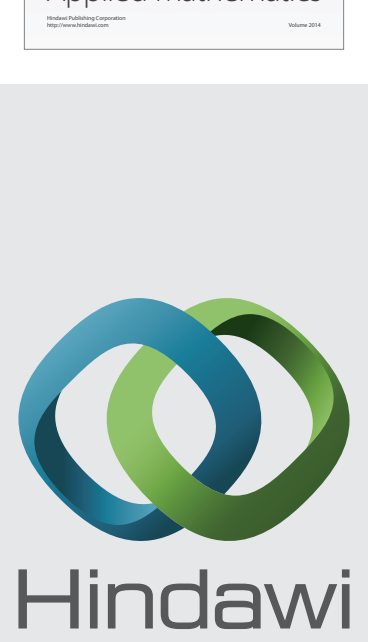

Submit your manuscripts at http://www.hindawi.com
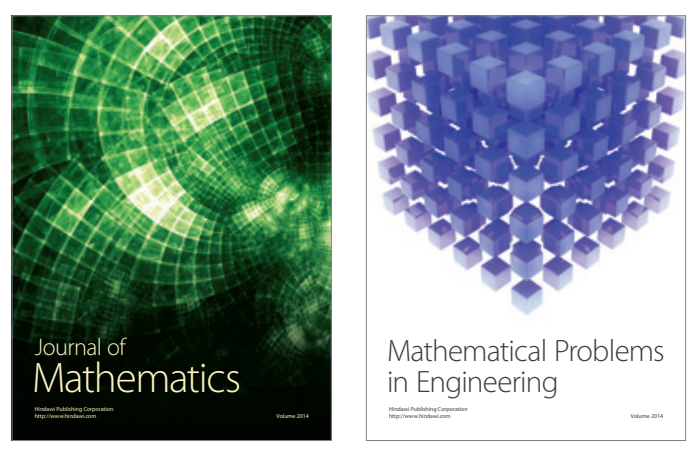

Mathematical Problems in Engineering
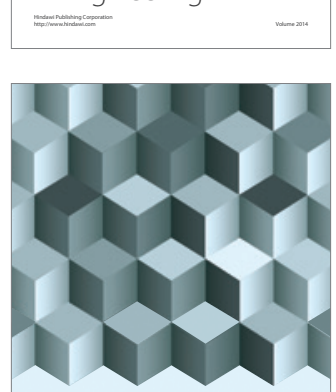

Journal of

Function Spaces
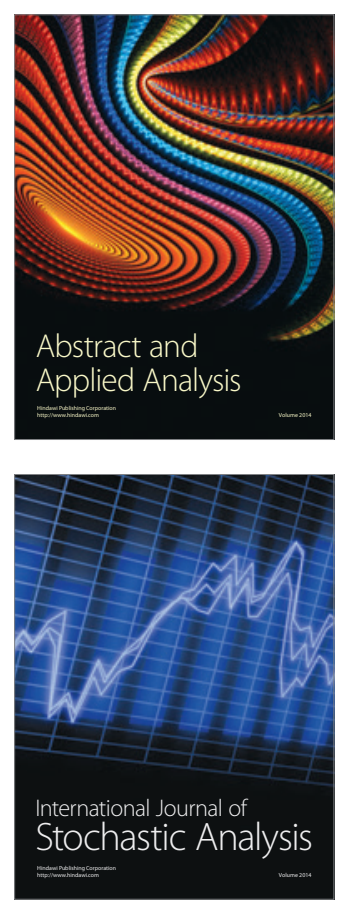

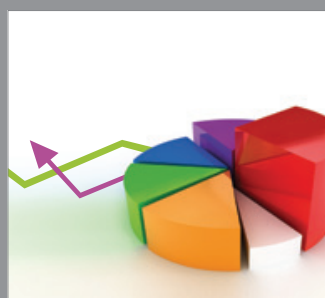

ournal of

Probability and Statistics

Promensencen
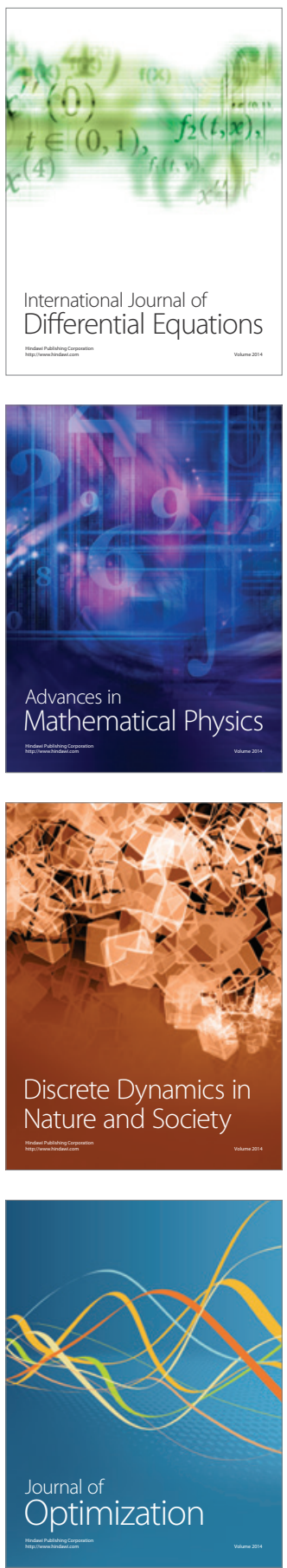